This postprint is published in:

Communications in analysis and geometry, Volume 28, Number 3, 2020, 677-706

DOI: 10.4310/CAG.2020.v28.n3.a6.

\title{
EXISTENCE RESULTS FOR SOME PROBLEMS ON RIEMANNIAN MANIFOLDS
}

\author{
GIOVANNI MOLICA BISCI, DUŠAN D. REPOVŠ, AND LUCA VILASI
}

\begin{abstract}
By using variational techniques we provide new existence results for Yamabetype equations with subcritical perturbations set on a compact $d$-dimensional $(d \geq 3)$ Riemannian manifold without boundary. As a direct consequence of our main theorems, we prove the existence of at least one solution to the following singular Yamabe-type problem

$$
\left\{\begin{array}{l}
-\Delta_{g} w+\alpha(\sigma) w=\mu K(\sigma) w^{\frac{d+2}{d-2}}+\lambda\left(w^{r-1}+f(w)\right), \quad \sigma \in \mathcal{M} \\
w \in H_{\alpha}^{2}(\mathcal{M}), \quad w>0 \text { in } \mathcal{M}
\end{array}\right.
$$

where, as usual, $\Delta_{g}$ denotes the Laplace-Beltrami operator on $(\mathcal{M}, g), \alpha, K: \mathcal{M} \rightarrow \mathbb{R}$ are positive (essentially) bounded functions, $r \in(0,1)$, and $f:[0,+\infty) \rightarrow[0,+\infty)$ is a subcritical continuous function. Restricting ourselves to the unit sphere $\mathbb{S}^{d}$ via the stereographic projection, we also solve some parametrized Emden-Fowler equations in the Euclidean case.
\end{abstract}

\section{INTRODUCTION}

In the present paper we explore the existence of solutions to the following problem

$$
\left(P_{\lambda, \mu}\right) \quad-\Delta_{g} w+\alpha(\sigma) w=\mu K(\sigma)|w|^{\frac{4}{d-2}} w+\lambda f(w), \quad \sigma \in \mathcal{M}, w \in H_{1}^{2}(\mathcal{M}),
$$

where $(\mathcal{M}, g)$ is a compact $d$-dimensional Riemannian manifold without boundary, $d \geq 3$, $\Delta_{g}$ is the Laplace-Beltrami operator on $(\mathcal{M}, g)$, expressed in local coordinates $\left(x_{1}, \ldots, x_{d}\right)$ by

$$
\Delta_{g} w:=g^{i j}\left(\frac{\partial^{2} w}{\partial x^{i} \partial x^{j}}-\Gamma_{i j}^{k} \frac{\partial w}{\partial x^{k}}\right)
$$

where $\Gamma_{i j}^{k}$ are the well-known Christoffel symbols, the functions $\alpha, K: \mathcal{M} \rightarrow \mathbb{R}$ belong to the class

$$
\Lambda_{+}(\mathcal{M}):=\left\{\varphi \in L^{\infty}(\mathcal{M}, \mathbb{R}): \underset{\sigma \in \mathcal{M}}{\operatorname{essinf}} \varphi(\sigma)>0\right\}
$$

$\lambda, \mu$ are positive real parameters, and $f: \mathbb{R} \rightarrow \mathbb{R}$ is a continuous function with subcritical growth.

Problem $\left(P_{\lambda, \mu}\right)$ displays the same structure as the Yamabe equation with the addition of a subcritical perturbation term. As is well-known, the latter equation is intimately related to the following problem arising in differential geometry.

Problem 1.1. Given a d-dimensional compact Riemannian manifold $(M, g), d>2$, with scalar curvature $k=k(\sigma)$, find a metric $\tilde{g}$ conformal to $g$, with constant scalar curvature $\tilde{k}$.

Key words and phrases. Yamabe equation, Riemannian manifold, Emden-Fowler equation, LaplaceBeltrami operator, existence of solutions

2010 AMS Subject Classification: Primary: 35A15, 35S15, 49J35; Secondary: 45G05, 47G20. 
Setting $\tilde{g}:=w^{4 /(d-2)} g$, where $w>0$ is the conformal factor, this requirement can be stated in terms of partial differential equations as follows:

Find $w \in C^{\infty}(\mathcal{M}), w>0$, satisfying

$$
-\Delta_{g} w+\frac{d-2}{4(d-1)} k(\sigma) w=\tilde{k} w^{\frac{d+2}{d-2}} \quad \text { in } \mathcal{M} .
$$

The history of equation (1.2) and attempts on its resolution spans more than two decades. In 1960, Yamabe [27] claimed to have found a solution but his proof turned out to be wrong and was repaired by Trudinger [25] for the case when the conformal class of the reference metric is nonpositive. Some years later, Aubin [2] provided a positive answer to the question for all manifolds of dimension $d \geq 6$ which are not conformally flat; the remaining and more difficult cases were definitively solved by Schoen [22].

Elliptic problems set on compact Riemannian manifolds without boundary, as well as their applications to Emden-Fowler equations, have been the object of recent study, especially in the sublinear case. In [16] Kristály and Rădulescu obtained multiple solutions to the problem

$$
-\Delta_{g} w+\alpha(\sigma) w=\mu K(\sigma) f(w), \quad \sigma \in \mathcal{M}, w \in H_{1}^{2}(\mathcal{M}),
$$

when $f$ has a sublinear behaviour at infinity. More precisely, in their Theorem 1.1 they proved the existence of two nontrivial solutions to $\left(P_{\mu}\right)$, for $\mu$ sufficiently large, through a careful analysis of the mountain pass geometry of the functional energy. Under some natural compactness assumptions, a similar approach applies to problem $\left(P_{\lambda, \mu}\right)$ as well, and leads to the existence of at least one nontrivial solution, as shown in our Theorem 4.1 (see Section 4).

However, here we are going to propose a different approach, quite new in the framework of elliptic equations on manifolds, which in particular does not use the celebrated Lions concentration-compactness principle (see [18]) or one of its many variants. As it is wellknown, the latter represents a major tool in the study of critical elliptic equations and has been intensively used in literature in many different contexts.

Our first existence result (Theorem 3.1) relies upon direct minimization techniques on small balls of the energy space. To be more precise, we prove that, for every $\mu>0$, the restriction of the functional

$$
\begin{aligned}
w \mapsto & \frac{1}{2} \int_{\mathcal{M}}\left(|\nabla w(\sigma)|^{2}+\alpha(\sigma) w(\sigma)^{2}\right) d \sigma_{g} \\
& -\frac{\mu}{2^{*}} \int_{\mathcal{M}} K(\sigma)|w(\sigma)|^{2^{*}} d \sigma_{g}, \quad w \in H_{\alpha}^{2}(\mathcal{M}),
\end{aligned}
$$

to a ball of $H_{\alpha}^{2}(\mathcal{M})$ centered at 0 and of small enough radius, is sequentially weakly lower semicontinuous (see Lemma 3.2).

Consequently, the whole energy functional associated with $\left(P_{\lambda, \mu}\right)$ is locally sequentially weakly lower semicontinuous and so it will admit, for any $\mu>0$ and sufficiently small $\lambda$, a local minimum. We point out that throughout our paper the nonlinearity $f$ is merely required to be subcritical and with an appropriate asymptotic behaviour at 0 . The key point is the smallness of $\lambda$, which needs to belong to the range of a suitable rational function, involving among other things the sharp constant of the continuous (but not compact) embedding $H_{\alpha}^{2}(\mathcal{M}) \hookrightarrow L^{2 d /(d-2)}(\mathcal{M})$, see Remark 2.1.

We furthermore emphasize that our approach, originally due to Chabrowski [6], has been previously used in literature for studying classical quasilinear $p$-Laplacian equations involving critical nonlinearities (see [10], by which this paper was inspired, and [23]). For 
the sake of completeness, we also mention [21], where the noncompact analogue of $\left(P_{\lambda, \mu}\right)$ was investigated, and the recent papers $[13,19,20]$ where, by adopting similar variational methods, the existence of weak solutions for nonlocal fractional equations and subelliptic problems on Carnot groups were studied in situations of lack of compactness.

Theorem 3.1 has connections with several results obtained in the case of bounded domains of $\mathbb{R}^{d}$, like the main one of [24], where Tarantello showed that the problem

$$
\left\{\begin{array}{l}
-\Delta u=|u|^{\frac{4}{d-2}} u+h(x) \quad \text { in } \Omega \\
u=0 \quad \text { on } \partial \Omega,
\end{array}\right.
$$

where $\Omega \subset \mathbb{R}^{d}, d>2$, and $h \in H^{-1}(\Omega)$, admits two distinct weak solutions provided that $h$ has a sufficiently small $\|\cdot\|_{-1,2}$-norm, namely

$$
\|h\|_{-1,2} \leq \frac{4}{d-2}\left(\frac{d-2}{d+2}\right)^{(d+2) / 4} S^{d / 4}
$$

where $S$ is the best critical Sobolev constant for the embedding $H_{0}^{1}(\Omega) \hookrightarrow L^{2^{*}}(\Omega)$.

There are other connections with the central result of [12], dealing with the perturbed critical elliptic problem

$$
\left\{\begin{array}{l}
-\Delta u=|u|^{\frac{4}{d-2}} u+g(x, u) \quad \text { in } \Omega \\
u=0 \quad \text { on } \partial \Omega
\end{array}\right.
$$

where $\Omega \subset \mathbb{R}^{d}, d \geq 3$, and the nonlinearity $g$ has a subcritical growth at infinity.

Among all cases covered by $\left(P_{\lambda, \mu}\right)$, a remarkable one is given by

$$
\begin{aligned}
- & \Delta_{h} w+s(1-s-d) w \\
& =\mu K(\sigma)|w|^{\frac{4}{d-2}} w+\lambda f(w), \quad \sigma \in \mathbb{S}^{d}, w \in H_{1}^{2}\left(\mathbb{S}^{d}\right),
\end{aligned}
$$

where $\mathbb{S}^{d}$ is the unit sphere in $\mathbb{R}^{d+1}, h$ is the standard metric induced by the embedding $\mathbb{S}^{d} \hookrightarrow \mathbb{R}^{d+1}, s$ is a constant such that $1-d<s<0$ and $\Delta_{h}$ denotes the Laplace-Beltrami operator on $\left(\mathbb{S}^{d}, h\right)$. It yields equation $(1.2)$ on $\mathbb{S}^{d}$ when $s=-d / 2$ or $s=-d / 2+1$ (see for instance $[3,7,8,14,15,26]$ and references therein for a wider framework on these topics).

Problem $\left(\widetilde{P}_{\lambda, \mu}\right)$ has an analogue in the Euclidean context: any existence result for $\left(\widetilde{P}_{\lambda, \mu}\right)$ indeed can be translated, by an appropriate change of coordinates, into an existence result for the following parametrized Emden-Fowler type equation,

$$
\begin{aligned}
-\Delta u= & \mu|x|^{\frac{2(2-2 s-d)}{d-2}} K\left(\frac{x}{|x|}\right)|u|^{\frac{4}{d-2}} u \\
& +\lambda|x|^{s-2} f\left(\frac{u}{|x|^{s}}\right), \quad x \in \mathbb{R}^{d+1} \backslash\{0\},
\end{aligned}
$$

see Section 5 for details. The latter represents a generalization of the more common (and unperturbed) equation

$$
-\Delta u=\mu|x|^{s-2} K\left(\frac{x}{|x|}\right) f\left(\frac{u}{|x|^{s}}\right), \quad x \in \mathbb{R}^{d+1} \backslash\{0\},
$$

which, in the presence of a pure superlinear nonlinearity (that is, for $f(t)=|t|^{p-1} t, p>1$ ), has been treated by minimization and minimax techniques in the classical papers [8] and $[26]$.

If the perturbation term in $\left(P_{\lambda, \mu}\right)$ is modified with the addition of a singular power at zero, then via the same approach as before (cf. also [10]), it is still possible to get nontrivial 
solutions to the singular Yamabe problem

$$
\left\{\begin{array}{l}
-\Delta_{g} w+\alpha(\sigma) w=\mu K(\sigma) w^{\frac{d+2}{d-2}}+\lambda\left(w^{r-1}+f(w)\right), \quad \sigma \in \mathcal{M} \\
w \in H_{\alpha}^{2}(\mathcal{M}), \quad w>0 \text { in } \mathcal{M},
\end{array}\right.
$$

where $r \in(0,1)$ and $f:[0,+\infty) \rightarrow[0,+\infty)$ is continuous and subcritical, see Theorem 3.7.

As a consequence, it is straightforward to deduce the existence of at least one nontrivial solution to the following singular equation

$$
\left\{\begin{array}{l}
-\Delta_{h} w+s(1-s-d) w=\mu K(\sigma) w^{\frac{d+2}{d-2}}+\lambda\left(w^{r-1}+f(w)\right), \quad \sigma \in \mathbb{S}^{d} \\
w \in H_{1}^{2}\left(\mathbb{S}^{d}\right), \quad w>0 \text { in } \mathbb{S}^{d}
\end{array}\right.
$$

and this is the content of our Theorem 5.3.

For the sake of completeness we cite related paper [11] in which the authors studied the existence of nonnegative solutions for the doubly critical equation of the form

$$
-\Delta_{p} u-\mu \frac{u^{p-1}}{|x|^{p}}=u^{p^{*}-1}+\frac{u^{p^{*}(s)-1}}{|x|^{s}}, \quad \text { in } \mathbb{R}^{d}
$$

where $\Delta_{p}$ is the usual $p$-Laplace operator (with $1<p<d$ and $d \geq 2$ ), $\mu$ is a real parameter, $p^{*}$ is the critical Sobolev exponent, $0<s<p$ and $p^{*}(s):=p(d-s) /(d-p)$. We also point out that a Lane-Emden-Fowler equation on a bounded Euclidean domain and involving a singular potential was studied in [9]. Furthermore, under appropriate spectral assumptions, two existence results for positive solutions of Lichnerowicz-type equations on complete (noncompact) manifolds were proved in [1].

The paper is organized as follows. In Section 2 we review some basic definitions and facts on Sobolev spaces defined on compact Riemannian manifolds, while in Section 3 we state and prove our main result of existence of solutions for $\left(P_{\lambda, \mu}\right)$. Section 4 is devoted to another existence result, obtained this time as an application of a version of the Mountain Pass Theorem without the Palais-Smale condition due to Brezis and Nirenberg ([5, Theorem $2.2])$ proving an existence result for problem $\left(P_{\lambda, \mu}\right)$. Some concrete applications of our general theorems to Emden-Fowler equations are illustrated in the last section.

\section{PRELIMINARIES AND VARIATIONAL FRAMEWORK}

In this section we recall some notions and basic facts on Sobolev spaces on compact Riemannian manifolds which will be helpful in the sequel. We refer the reader to $[3,14]$ for detailed derivations of the geometric quantities, their motivation and further applications. We also mention recent monograph [17] as a general reference on this subject.

Let $(\mathcal{M}, g)$ be a smooth compact $d$-dimensional Riemannian manifold, $d \geq 3$, without boundary and let $g_{i j}$ be the components of the metric $g$. If $\alpha \in \Lambda_{+}(\mathcal{M})$ (see (1.1)) and $C^{\infty}(\mathcal{M})$ denotes as usual the space of smooth functions defined on $\mathcal{M}$, we set

$$
\|w\|_{H_{\alpha}^{2}}:=\left(\int_{\mathcal{M}}|\nabla w(\sigma)|^{2} d \sigma_{g}+\int_{\mathcal{M}} \alpha(\sigma) w(\sigma)^{2} d \sigma_{g}\right)^{1 / 2},
$$

for every $w \in C^{\infty}(\mathcal{M})$, where $\nabla w$ is the covariant derivative of $w$ and $d \sigma_{g}$ is the Riemannian measure on $\mathcal{M}$. In terms of local coordinates $\left(x^{1}, \ldots, x^{d}\right), \nabla w$ can be represented by

where

$$
\left(\nabla^{2} w\right)_{i j}=\frac{\partial^{2} w}{\partial x^{i} \partial x^{j}}-\Gamma_{i j}^{k} \frac{\partial w}{\partial x^{k}}
$$

$$
\Gamma_{i j}^{k}:=\frac{1}{2}\left(\frac{\partial g_{l j}}{\partial x^{i}}+\frac{\partial g_{l i}}{\partial x^{j}}-\frac{\partial g_{i j}}{\partial x^{k}}\right) g^{l k}
$$


are the usual Christoffel symbols and $g^{l k}$ are the elements of the inverse matrix of $g$, Einstein's summation convention being tacitly adopted.

The Riemannian volume element $d \sigma_{g}$ in (2.1) is given by $d \sigma_{g}=$ $(\operatorname{det} g)^{1 / 2} d x$, where $d x$ stands for the Lebesgue volume element of $\mathbb{R}^{d}$, and we set

$$
\operatorname{Vol}_{g}(\mathcal{M}):=\int_{\mathcal{M}} d \sigma_{g}
$$

In the special case $(\mathcal{M}, g)=\left(\mathbb{S}^{d}, h\right)$, where $\mathbb{S}^{d}$ is the unit sphere in $\mathbb{R}^{d+1}$ and $h$ is the standard metric induced by the embedding $\mathbb{S}^{d} \hookrightarrow \mathbb{R}^{d+1}$, we use the notation

$$
\omega_{d}:=\operatorname{Vol}_{h}\left(\mathbb{S}^{d}\right)=\int_{\mathbb{S}^{d}} d \sigma_{h} .
$$

The Sobolev space $H_{\alpha}^{2}(\mathcal{M})$ is defined as the completion of $C^{\infty}(\mathcal{M})$ with respect to the norm $\|\cdot\|_{H_{\alpha}^{2}}$. Such a space turns out to be a Hilbert space when endowed with the inner product

$$
\begin{aligned}
\left\langle w_{1}, w_{2}\right\rangle_{H_{\alpha}^{2}}= & \int_{\mathcal{M}}\left\langle\nabla w_{1}(\sigma), \nabla w_{2}(\sigma)\right\rangle_{g} d \sigma_{g} \\
& +\int_{\mathcal{M}} \alpha(\sigma)\left\langle w_{1}(\sigma), w_{2}(\sigma)\right\rangle_{g} d \sigma_{g}
\end{aligned}
$$

for every $w_{1}, w_{2} \in H_{\alpha}^{2}(\mathcal{M})$, where $\langle\cdot, \cdot\rangle_{g}$ is the inner product on covariant tensor fields associated with $g$.

Because of the positivity of $\alpha,\|\cdot\|_{H_{\alpha}^{2}}$ is equivalent to the standard norm

$$
\|w\|_{H_{1}^{2}}=\left(\int_{\mathcal{M}}|\nabla w(\sigma)|^{2} d \sigma_{g}+\int_{\mathcal{M}} w(\sigma)^{2} d \sigma_{g}\right)^{1 / 2},
$$

as is plainly deducible from the following inequalities

$$
\min \left\{1, \underset{\sigma \in \mathcal{M}}{\operatorname{essinf}} \alpha(\sigma)^{1 / 2}\right\}\|w\|_{H_{1}^{2}} \leq\|w\|_{H_{\alpha}^{2}} \leq \max \left\{1,\|\alpha\|_{\infty}^{1 / 2}\right\}\|w\|_{H_{1}^{2}},
$$

which hold for any $w \in H_{\alpha}^{2}(\mathcal{M})$. Hereafter, we shall drop the subscripts in $(2.1)$ and $(2.2)$ and denote $\|\cdot\|_{H_{\alpha}^{2}}$ and $\langle\cdot, \cdot\rangle_{H_{\alpha}^{2}}$ simply by $\|\cdot\|$ and $\langle\cdot, \cdot\rangle$, respectively.

On account of Rellich-Kondrachov's theorem for compact manifolds without boundary, one has

$$
H_{\alpha}^{2}(\mathcal{M}) \hookrightarrow L^{q}(\mathcal{M}) \quad \text { for every } q \in\left[1,2^{*}\right]
$$

where

$$
2^{*}=\frac{2 d}{d-2},
$$

and the above embedding is also compact whenever $q \in\left[1,2^{*}\right)$. For any $q \in\left[1,2^{*}\right]$ we denote by $\|\cdot\|_{q}$ the standard $L^{q}$-norm on $\mathcal{M}$ and by $c_{q}$ the best constant of (2.5), i.e.

$$
c_{q}:=\sup _{w \in H_{\alpha}^{2}(\mathcal{M}) \backslash\{0\}} \frac{\|w\|_{q}}{\|w\|}>0,
$$

while we reserve the symbol $S$ for the positive constant

$$
S:=\inf _{w \in H_{\alpha}^{2}(\mathcal{M}) \backslash\{0\}} \frac{\|w\|^{2}}{\|w\|_{2^{*}}^{2}} .
$$


The open (respectively, closed) ball centered at $w \in H_{\alpha}^{2}(\mathcal{M})$ of radius $r>0$ will be denoted by $B(w, r)$ (respectively, $B_{c}(u, r)$ ), and the sphere $\left\{z \in H_{\alpha}^{2}(\mathcal{M}):\|w-z\|=r\right\}$ by $\partial B(w, r)$.

In what follows, we shall make the standing assumptions that $K \in \Lambda_{+}(\mathcal{M})$ and $f: \mathbb{R} \rightarrow \mathbb{R}$ is locally Lipschitz continuous. It is straightforward to check that problem $\left(P_{\lambda, \mu}\right)$ represents the Euler-Lagrange equation of the functional

$$
\begin{aligned}
\mathcal{E}_{\lambda, \mu}(w):= & \frac{1}{2}\|w\|^{2}-\frac{\mu}{2^{*}} \int_{\mathcal{M}} K(\sigma)|w|^{2^{*}} d \sigma_{g} \\
& -\lambda \int_{\mathcal{M}} F(w(\sigma)) d \sigma_{g}, \quad w \in H_{\alpha}^{2}(\mathcal{M}),
\end{aligned}
$$

where

$$
F(t):=\int_{0}^{t} f(\tau) d \tau \quad \text { for all } t \in \mathbb{R} .
$$

The critical points $w$ of $\mathcal{E}_{\lambda_{\mu}}$ are therefore the weak solutions to $\left(P_{\lambda, \mu}\right)$, i.e. they satisfy the relationship

$$
\langle w, z\rangle=\mu \int_{\mathcal{M}} K(\sigma)|w|^{\frac{4}{d-2}} w z d \sigma_{g}+\lambda \int_{\mathcal{M}} f(w) z d \sigma_{g},
$$

for all $z \in H_{\alpha}^{2}(\mathcal{M})$ and, furthermore, the regularity assumptions on $K$ and $f$ force any weak solution to be actually a classical one.

It is worth observing that, fixing $\lambda, \mu \in \mathbb{R}$, the constant function $w(\sigma)=c \in \mathbb{R}$ is a (trivial) solution to $\left(P_{\lambda, \mu}\right)$ if and only if

$$
c=\frac{\mu K(\sigma)|c|^{\frac{4}{d-2}} c+\lambda f(c)}{\alpha(\sigma)} \quad \text { in } \mathcal{M}
$$

Thus, constant solutions to $\left(P_{\lambda, \mu}\right)$ appear as fixed points of the real function

$$
t \mapsto \frac{\mu K(\sigma)|t|^{\frac{4}{d-2}} t+\lambda f(t)}{\alpha(\sigma)}, \quad t \in \mathbb{R} .
$$

Remark 2.1. It is well-known that sharp Sobolev inequalities play a crucial role in the theory partial differential equations, from both theoretical and applied point of view, and there is a broad literature on this subject. In our context, when $(\mathcal{M}, g)=\left(\mathbb{S}^{d}, h\right)$, a concrete upper bound for the constants $c_{q}, q \in[1,2 d /(d-2))$, depending on the geometry of $\mathbb{S}^{d}$, can be obtained as

$$
c_{q} \leq \frac{\kappa_{q}}{\min \left\{1, \min _{\sigma \in \mathbb{S}^{d}} \alpha(\sigma)^{1 / 2}\right\}},
$$

where

$$
\kappa_{q}:= \begin{cases}\omega_{d}^{\frac{2-q}{2 q}} & \text { if } q \in[1,2), \\ \max \left\{\left(\frac{q-2}{d} \omega_{d}^{\frac{2-q}{q}}\right)^{1 / 2}, \omega_{d}^{\frac{2-q}{2 q}}\right\} & \text { if } q \in\left[2, \frac{2 d}{d-2}\right) .\end{cases}
$$

Indeed, for every $2 \leq q<2 d /(d-2)$ and $w \in H_{1}^{2}\left(\mathbb{S}^{d}\right)$, one has

$$
\left(\int_{\mathbb{S}^{d}}|w(\sigma)|^{q} d \sigma_{h}\right)^{2 / q} \leq \frac{q-2}{d \omega_{d}^{1-2 / q}} \int_{\mathbb{S}^{d}}|\nabla w(\sigma)|^{2} d \sigma_{h}+\frac{1}{\omega_{d}^{1-2 / q}} \int_{\mathbb{S}^{d}}|w(\sigma)|^{2} d \sigma_{h}
$$


(cf. for instance [4] and [14]) and thus

$$
\begin{aligned}
\|w\|_{q} \leq & \max \left\{\left(\frac{q-2}{d} \omega_{d}^{\frac{2-q}{q}}\right)^{1 / 2}, \omega_{d}^{\frac{2-q}{2 q}}\right\} \\
& \times\left(\int_{\mathbb{S}^{d}}|\nabla w(\sigma)|^{2} d \sigma_{h}+\int_{\mathbb{S}^{d}}|w(\sigma)|^{2} d \sigma_{h}\right)^{1 / 2},
\end{aligned}
$$

which, by (2.4), implies the desired estimate. On the other hand, if $q \in[1,2)$, it follows from Hölder's inequality that

$$
\|w\|_{q} \leq \omega_{d}^{\frac{2-q}{2 q}}\|w\|_{2}, \quad \text { for all } w \in L^{2}\left(\mathbb{S}^{d}\right)
$$

and the conclusion is achieved by taking into account that

$$
\|w\|_{2} \leq\|w\|_{H_{1}^{2}\left(\mathbb{S}^{d}\right)} \leq \frac{\|w\|}{\min \left\{1, \min _{\sigma \in \mathbb{S}^{d}} \alpha(\sigma)^{1 / 2}\right\}}
$$

for every $w \in H_{1}^{2}\left(\mathbb{S}^{d}\right)$.

\section{EXISTENCE OF LOCAL MINIMIZERS}

Our first existence result for problem $\left(P_{\lambda, \mu}\right)$ can be stated as follows:

Theorem 3.1. Let $f: \mathbb{R} \rightarrow \mathbb{R}$ satisfy the following requirements:

$\left(f_{1}\right)$ there exist $a_{1}, a_{2}>0$ and $q \in\left[1,2^{*}\right)$ such that

$$
|f(t)| \leq a_{1}+a_{2}|t|^{q-1} \quad \text { for all } t \in \mathbb{R}
$$

$\left(f_{2}\right) \liminf _{t \rightarrow 0^{+}} \frac{F(t)}{t^{2}}=+\infty$.

Furthermore, for any $\mu>0$ let $l_{\mu}:[0,+\infty) \rightarrow \mathbb{R}$ be the function defined by

$$
l_{\mu}(t):=\frac{t-\mu c_{2^{*}}^{2^{*}}\|K\|_{\infty} t^{2^{*}-1}}{a_{1} c_{2^{*}} \operatorname{Vol}_{g}(\mathcal{M})^{\frac{2^{*}-1}{2^{*}}}+a_{2} c_{2^{*}}^{q} \operatorname{Vol}_{g}(\mathcal{M})^{\frac{2^{*}-q}{2^{*}}} t^{q-1}} \quad \text { for all } t \geq 0 .
$$

Then for every $\mu>0$ there exists an open interval

$$
\Lambda_{\mu} \subseteq\left(0, \max _{[0,+\infty)} l_{\mu}\right)
$$

such that, for every $\lambda \in \Lambda_{\mu},\left(P_{\lambda, \mu}\right)$ admits a nontrivial solution $w_{0, \mu, \lambda} \in H_{\alpha}^{2}(\mathcal{M})$.

As already mentioned, our strategy consists in showing that the energy (2.7) possesses a nontrivial minimizer in $H_{\alpha}^{2}(\mathcal{M})$. Yet, the presence of the critical term prevents the direct minimization from being immediately applicable. First we consider, for all $w \in H_{\alpha}^{2}(\mathcal{M})$, the functionals

$$
w \mapsto \mathcal{E}_{\lambda, \mu}(w)+\lambda \int_{\mathcal{M}} F(w) d \sigma_{g}, \quad w \mapsto \frac{1}{2}\|w\|^{2}-\mathcal{E}_{\lambda, \mu}(w),
$$

and prove some of their properties.

Lemma 3.2. For every $\mu>0$ there exists $\varrho_{0, \mu}>0$ such that the functional

$$
\widehat{\mathcal{E}}_{\mu}(w):=\frac{1}{2}\|w\|^{2}-\frac{\mu}{2^{*}} \int_{\mathcal{M}} K(\sigma)|w(\sigma)|^{2^{*}} d \sigma_{g}, \quad w \in H_{\alpha}^{2}(\mathcal{M}),
$$

is sequentially weakly lower semicontinuous in $B_{c}\left(0, \varrho_{0, \mu}\right)$. 
Proof. Let $\mu, \varrho>0$ and let $\left\{w_{j}\right\}_{j \in \mathbb{N}} \subset B_{c}(0, \varrho)$ be such that $w_{j} \rightarrow w_{\infty} \in B_{c}(0, \varrho)$. The conclusion will be achieved by proving that

$$
\liminf _{j \rightarrow \infty}\left(\widehat{\mathcal{E}}_{\mu}\left(w_{j}\right)-\widehat{\mathcal{E}}_{\mu}\left(w_{\infty}\right)\right) \geq 0 .
$$

Let us observe that, for all $w_{1}, w_{2} \in H_{\alpha}^{2}(\mathcal{M})$, the following basic equality holds

$$
\left\|w_{2}\right\|^{2}-\left\|w_{1}\right\|^{2}-2\left\langle w_{1}, w_{2}-w_{1}\right\rangle_{g}=\left\|w_{1}-w_{2}\right\|^{2} .
$$

Moreover, applying Brézis-Lieb's Lemma to the sequence $\left\{K^{1 / 2^{*}} w_{j}\right\}_{j \in \mathbb{N}} \subset L^{2^{*}}(\mathcal{M})$, one has

$$
\begin{aligned}
& \liminf _{j \rightarrow \infty}\left(\int_{\mathcal{M}} K(\sigma)\left|w_{j}(\sigma)\right|^{2^{*}} d \sigma_{g}-\int_{\mathcal{M}} K(\sigma)\left|w_{\infty}(\sigma)\right|^{2^{*}} d \sigma_{g}\right) \\
& =\liminf _{j \rightarrow \infty} \int_{\mathcal{M}} K(\sigma)\left|w_{j}(\sigma)-w_{\infty}(\sigma)\right|^{2^{*}} d \sigma_{g} .
\end{aligned}
$$

Bearing in mind also that $w_{j} \rightarrow w_{\infty}$, we deduce

$$
\begin{aligned}
& \liminf _{j \rightarrow \infty}\left(\widehat{\mathcal{E}}_{\mu}\left(w_{j}\right)-\widehat{\mathcal{E}}_{\mu}\left(w_{\infty}\right)\right) \\
= & \liminf _{j \rightarrow \infty}\left(\frac{1}{2}\left(\left\|w_{j}\right\|^{2}-\left\|w_{\infty}\right\|^{2}\right)\right. \\
& \left.-\frac{\mu}{2^{*}} \int_{\mathcal{M}} K(\sigma)\left(\left|w_{j}(\sigma)\right|^{2^{*}}-\left|w_{\infty}(\sigma)\right|^{2^{*}}\right) d \sigma_{g}\right) \\
\geq & \liminf _{j \rightarrow \infty}\left(\frac{1}{2}\left\|w_{j}-w_{\infty}\right\|^{2}-\frac{\mu}{2^{*}}\|K\|_{\infty} \int_{\mathcal{M}}\left|w_{j}(\sigma)-w_{\infty}(\sigma)\right|^{2^{*}} d \sigma_{g}\right) \\
\geq & \liminf _{j \rightarrow \infty}\left(\frac{1}{2}-\frac{c_{2^{*}}^{2^{*}}}{2^{*}} \mu K\left\|_{\infty}\right\| w_{j}-w_{\infty} \|^{2^{*}-2}\right)\left\|w_{j}-w_{\infty}\right\|^{2} \\
\geq & \liminf _{j \rightarrow \infty}\left(\frac{1}{2}-\frac{c_{2^{*}}^{2^{*}}}{2^{*}} \mu\|K\|_{\infty} \varrho^{2^{*}-2}\right)\left\|w_{j}-w_{\infty}\right\|^{2} .
\end{aligned}
$$

So for

$$
0<\varrho \leq \bar{\varrho}_{\mu}:=\left(\frac{d}{(d-2) c_{2^{*}}^{2^{*}} \mu K \|_{\infty}}\right)^{\frac{d-2}{4}},
$$

inequality (3.2) is verified and $\widehat{\mathcal{E}}_{\mu}$ is sequentially weakly lower semicontinuous in $B_{c}\left(0, \varrho_{0, \mu}\right)$, provided that $\varrho_{0, \mu} \in\left(0, \varrho_{\mu}\right)$.

Lemma 3.3. Let $\lambda, \mu>0$, let $f: \mathbb{R} \rightarrow \mathbb{R}$ satisfy $\left(f_{1}\right)$, and let $\widetilde{\mathcal{E}}_{\lambda, \mu}: H_{\alpha}^{2}(\mathcal{M}) \rightarrow \mathbb{R}$ be the functional defined by

$$
\widetilde{\mathcal{E}}_{\lambda, \mu}(w):=\frac{\mu}{2^{*}} \int_{\mathcal{M}} K(\sigma)|w(\sigma)|^{2^{*}} d \sigma_{g}+\lambda \int_{\mathcal{M}} F(w) d \sigma_{g}
$$

for any $w \in H_{\alpha}^{2}(\mathcal{M})$. Then the following facts hold:

(i) if

$$
\limsup _{\varepsilon \rightarrow 0^{+}} \frac{\sup _{B_{c}\left(0, \varrho_{0}\right)} \widetilde{\mathcal{E}}_{\lambda, \mu}-\sup _{B_{c}\left(0, \varrho_{0}-\epsilon\right)} \widetilde{\mathcal{E}}_{\lambda, \mu}}{\varepsilon}<\varrho_{0}
$$

for some $\varrho_{0}>0$, then

$$
\inf _{\eta<\varrho_{0}} \frac{\sup _{B_{c}\left(0, \varrho_{0}\right)} \widetilde{\mathcal{E}}_{\lambda, \mu}-\sup _{B_{c}(0, \eta)} \widetilde{\mathcal{E}}_{\lambda, \mu}}{\varrho_{0}^{2}-\eta^{2}}<\frac{1}{2}
$$


(ii) if (3.5) is satisfied for some $\varrho_{0}>0$, then

$$
\inf _{w \in B\left(0, \varrho_{0}\right)} \frac{\sup _{B_{c}\left(0, \varrho_{0}\right)} \widetilde{\mathcal{E}}_{\lambda, \mu}-\widetilde{\mathcal{E}}_{\lambda, \mu}(w)}{\varrho_{0}^{2}-\|w\|^{2}}<\frac{1}{2} .
$$

Proof. (i) From the identity

$$
\frac{\sup _{B_{c}\left(0, \varrho_{0}\right)} \widetilde{\mathcal{E}}_{\lambda, \mu}-\sup _{B_{c}\left(0, \varrho_{0}-\epsilon\right)} \widetilde{\mathcal{E}}_{\lambda, \mu}}{\varrho_{0}^{2}-\left(\varrho_{0}-\varepsilon\right)^{2}}=\left(\frac{\sup _{B_{c}\left(0, \varrho_{0}\right)} \widetilde{\mathcal{E}}_{\lambda, \mu}-\sup _{B_{c}\left(0, \varrho_{0}-\epsilon\right)} \widetilde{\mathcal{E}}_{\lambda, \mu}}{\varepsilon}\right)\left(\frac{1}{2 \varrho_{0}-\varepsilon}\right),
$$

it follows that

$$
\limsup _{\varepsilon \rightarrow 0^{+}} \frac{\sup _{B_{c}\left(0, \varrho_{0}\right)} \widetilde{\mathcal{E}}_{\lambda, \mu}-\sup _{B_{c}\left(0, \varrho_{0}-\epsilon\right)} \widetilde{\mathcal{E}}_{\lambda, \mu}}{\varrho_{0}^{2}-\left(\varrho_{0}-\varepsilon\right)^{2}}<\frac{1}{2} .
$$

Now, by (3.7) there exists $\bar{\varepsilon}_{0}>0$ such that

$$
\frac{\sup _{B_{c}\left(0, \varrho_{0}\right)} \widetilde{\mathcal{E}}_{\lambda, \mu}-\sup _{B_{c}\left(0, \varrho_{0}-\varepsilon\right)} \widetilde{\mathcal{E}}_{\lambda, \mu}}{\varrho_{0}^{2}-\left(\varrho_{0}-\varepsilon\right)^{2}}<\frac{1}{2}
$$

for every $\varepsilon \in\left(0, \bar{\varepsilon}_{0}\right)$. Setting $\eta_{0}:=\varrho_{0}-\varepsilon_{0}$, with $\varepsilon_{0} \in\left(0, \bar{\varepsilon}_{0}\right)$, we get

$$
\frac{\sup _{B_{c}\left(0, \varrho_{0}\right)} \widetilde{\mathcal{E}}_{\lambda, \mu}-\sup _{B_{c}\left(0, \eta_{0}\right)} \widetilde{\mathcal{E}}_{\lambda, \mu}}{\varrho_{0}^{2}-\eta_{0}^{2}}<\frac{1}{2}
$$

and thus the conclusion follows.

(ii) Thanks to inequality (3.5), one has

$$
\sup _{B_{c}\left(0, \eta_{0}\right)} \widetilde{\mathcal{E}}_{\lambda, \mu}>\sup _{B_{c}\left(0, \varrho_{0}\right)} \widetilde{\mathcal{E}}_{\lambda, \mu}-\frac{1}{2}\left(\varrho_{0}^{2}-\eta_{0}^{2}\right)
$$

for some $0<\eta_{0}<\varrho_{0}$. Invoking $\left(f_{1}\right)$ and standard argument, $\widetilde{\mathcal{E}}_{\lambda, \mu}$ turns out to be weakly lower semicontinuous in $B_{c}\left(0, \eta_{0}\right)$ and therefore

$$
\sup _{\partial B_{c}\left(0, \eta_{0}\right)} \widetilde{\mathcal{E}}_{\lambda, \mu}=\sup _{\frac{B_{c}\left(0, \eta_{0}\right)}{*}} \widetilde{\mathcal{E}}_{\lambda, \mu}=\sup _{B_{c}\left(0, \eta_{0}\right)} \widetilde{\mathcal{E}}_{\lambda, \mu}
$$

where ${\overline{\partial B_{c}\left(0, \eta_{0}\right)}}^{*}$ is the weak closure of $\partial B_{c}\left(0, \eta_{0}\right)$ in $H_{\alpha}^{2}(\mathcal{M})$. Therefore by (3.8) there exists $w_{0} \in H_{\alpha}^{2}(\mathcal{M})$ with $\left\|w_{0}\right\|=\eta_{0}$ such that

$$
\widetilde{\mathcal{E}}_{\lambda, \mu}\left(w_{0}\right)>\sup _{B_{c}\left(0, \varrho_{0}\right)} \widetilde{\mathcal{E}}_{\lambda, \mu}-\frac{1}{2}\left(\varrho_{0}^{2}-\eta_{0}^{2}\right),
$$

and the second claim is proved as well.

We are now in a position to prove our existence result.

Proof of Theorem 3.1. Fix $\mu>0$ and let $\varrho_{\mu, \max }>0$ be the global maximizer of $l_{\mu}$. Set $\varrho_{0, \mu}:=\min \left\{\bar{\varrho}_{\mu}, \varrho_{\mu, \max }\right\}, \varrho_{\mu}$ being defined by $(3)$, and $\Lambda_{\mu}:=\left(0, l_{\mu}\left(\varrho_{0, \mu}\right)\right)$.

Taking $\lambda \in \Lambda_{\mu}$, there exists $\varrho_{0, \mu, \lambda} \in\left(0, \varrho_{0, \mu}\right)$ such that

$$
0<\lambda<\frac{\varrho_{0, \mu, \lambda}-\mu c_{2^{*}}^{2^{*}}\|K\|_{\infty} \varrho_{0, \mu, \lambda}^{2^{*}-1}}{a_{1} c_{2^{*}} \operatorname{Vol}_{g}(\mathcal{M})^{\frac{2^{*}-1}{2^{*}}}+a_{2} c_{2^{*}}^{q} \operatorname{Vol}_{g}(\mathcal{M})^{\frac{2^{*}-q}{2^{*}}} \varrho_{0, \mu, \lambda}^{q-1}} .
$$


Since $\varrho_{0, \mu, \lambda}<\varrho_{\mu}$, by Lemma 3.2 the functional $\mathcal{E}_{\lambda, \mu}$ is sequentially weakly lower semicontinuous in $B_{c}\left(0, \varrho_{0, \mu, \lambda}\right)$ and so there exists $w_{0, \mu, \lambda} \in$ $B_{c}\left(0, \varrho_{0, \mu, \lambda}\right)$ such that

$$
\mathcal{E}_{\lambda, \mu}\left(w_{0, \mu, \lambda}\right)=\min _{B_{c}\left(0, \varrho_{0, \mu, \lambda}\right)} \mathcal{E}_{\lambda, \mu}
$$

Suppose by contradiction that $\left\|w_{0, \mu, \lambda}\right\|=\varrho_{0, \mu, \lambda}$. Fix $\varepsilon \in\left(0, \varrho_{0, \mu, \lambda}\right)$ and define

$$
\varphi_{\lambda, \mu}\left(\varepsilon, \varrho_{0, \mu, \lambda}\right):=\frac{\sup _{B_{c}\left(0, \varrho_{0, \mu, \lambda}\right)} \widetilde{\mathcal{E}}_{\lambda, \mu}-\sup _{B_{c}\left(0, \varrho_{0, \mu, \lambda}-\varepsilon\right)} \widetilde{\mathcal{E}}_{\lambda, \mu}}{\varepsilon} .
$$

With the aid of $\left(f_{1}\right)$ we get

$$
\begin{aligned}
& \varphi_{\lambda, \mu}\left(\varepsilon, \varrho_{0, \mu, \lambda}\right) \\
\leq & \frac{1}{\varepsilon} \sup _{w \in B_{c}(0,1)} \int_{\mathcal{M}}\left|\int_{\left(\varrho_{0, \mu, \lambda}-\varepsilon\right) w(\sigma)}^{\varrho_{0, \mu, \lambda} w(\sigma)}\left(\mu\|K\|_{\infty}|t|^{2^{*}-1}+\lambda|f(t)|\right) d t\right| d \sigma_{g} \\
\leq & \frac{1}{\varepsilon} \sup _{w \in B_{c}(0,1)} \int_{\mathcal{M}}\left|\int_{\left(\varrho_{0, \mu, \lambda}-\varepsilon\right) w(\sigma)}^{\varrho_{0, \mu, \lambda} w(\sigma)}\left(\mu\|K\|_{\infty}|t|^{2^{*}-1}+a_{1} \lambda+a_{2} \lambda|t|^{q-1}\right) d t\right| d \sigma_{g} \\
\leq & \frac{c_{2^{*}}^{2^{*}} \mu\|K\|_{\infty}}{2^{*}}\left(\frac{\varrho_{0, \mu, \lambda}^{2^{*}}-\left(\varrho_{0, \mu, \lambda}-\varepsilon\right)^{2^{*}}}{\varepsilon}\right)+a_{1} \lambda c_{2^{*}} V o l_{g}(\mathcal{M})^{\frac{2^{*}-1}{2^{*}}} \\
& +a_{2} \lambda \frac{c_{2^{*}}^{q}}{q} \operatorname{Vol}_{g}(\mathcal{M})^{\frac{2^{*}-q}{2^{*}}}\left(\frac{\varrho_{0, \mu, \lambda}^{q}-\left(\varrho_{0, \mu, \lambda}-\varepsilon\right)^{q}}{\varepsilon}\right)
\end{aligned}
$$

and taking the limsup for $\varepsilon \rightarrow 0^{+}$we get

$$
\begin{aligned}
\limsup _{\varepsilon \rightarrow 0^{+}} \varphi_{\lambda, \mu}\left(\varepsilon, \varrho_{0, \mu, \lambda}\right) \leq & c_{2^{*}}^{2^{*}}\|K\|_{\infty} \varrho_{0, \mu, \lambda}^{2^{*}-1}+\lambda a_{1} c_{2^{*}} \operatorname{Vol} g(\mathcal{M})^{\frac{2^{*}-1}{2^{*}}} \\
& +\lambda a_{2} c_{2^{*}}^{q} \operatorname{Vol}_{g}(\mathcal{M})^{\frac{2^{*}-q}{2^{*}}} \varrho_{0, \mu, \lambda}^{q-1},
\end{aligned}
$$

which, due to (3.9), forces

$$
\limsup _{\varepsilon \rightarrow 0^{+}} \varphi_{\lambda, \mu}\left(\varepsilon, \varrho_{0, \mu, \lambda}\right)<\varrho_{0, \mu, \lambda} .
$$

Therefore, invoking Lemma 3.3, one has

$$
\inf _{w \in B\left(0, \varrho_{0, \mu, \lambda}\right)} \frac{\sup _{B_{c}\left(0, \varrho_{0, \mu, \lambda}\right)} \widetilde{\mathcal{E}}_{\lambda, \mu}-\widetilde{\mathcal{E}}_{\lambda, \mu}(w)}{\varrho_{0, \mu, \lambda}^{2}-\|w\|^{2}}<\frac{1}{2}
$$

and there exists $\bar{w}_{\mu, \lambda} \in B\left(0, \varrho_{0, \mu, \lambda}\right)$ such that, for every $w \in B_{c}\left(0, \varrho_{0, \mu, \lambda}\right)$,

$$
\widetilde{\mathcal{E}}_{\lambda, \mu}(w) \leq \sup _{B_{c}\left(0, \varrho_{0, \mu, \lambda}\right)} \widetilde{\mathcal{E}}_{\lambda, \mu}<\widetilde{\mathcal{E}}_{\lambda, \mu}\left(\bar{w}_{\mu, \lambda}\right)+\frac{1}{2}\left(\varrho_{0, \mu, \lambda}^{2}-\left\|\bar{w}_{\mu, \lambda}\right\|^{2}\right),
$$

which we can rewrite as

$$
\mathcal{E}_{\lambda, \mu}\left(\bar{w}_{\mu, \lambda}\right):=\frac{1}{2}\left\|\bar{w}_{\mu, \lambda}\right\|^{2}-\widetilde{\mathcal{E}}_{\lambda, \mu}\left(\bar{w}_{\mu, \lambda}\right)<\frac{\varrho_{0, \mu, \lambda}^{2}}{2}-\widetilde{\mathcal{E}}_{\lambda, \mu}(w) .
$$

Evaluating the previous inequality at $w=w_{0}$, we deduce

$$
\mathcal{E}_{\lambda, \mu}\left(\bar{w}_{\mu, \lambda}\right)<\frac{1}{2} \varrho_{0, \mu, \lambda}^{2}-\widetilde{\mathcal{E}}_{\lambda, \mu}\left(w_{0, \mu, \lambda}\right)=\mathcal{E}_{\lambda, \mu}\left(w_{0, \mu, \lambda}\right),
$$

against the minimality of $w_{0, \mu, \lambda}$. In conclusion, $w_{0, \mu, \lambda} \in B\left(0, \varrho_{0, \mu, \lambda}\right)$ and is therefore a local minimum for $\mathcal{E}_{\lambda, \mu}$ and a solution to $\left(P_{\lambda, \mu}\right)$. 
The final task is now to show that $w_{0, \mu, \lambda}$ is not identically 0 on $\mathcal{M}$. To this end, fix a constant $a \in(0,+\infty)$. Thanks to $\left(f_{2}\right)$, for all $c>0$ there exists $\delta_{c}>0$ such that

$$
F(t) \geq c t^{2} \quad \text { for any } t \in\left(0, \delta_{c}\right) .
$$

So if $t \in\left(0, \delta_{c} / a\right)$, we obtain

$$
\begin{aligned}
\mathcal{E}_{\lambda, \mu}(t a) & =\frac{1}{2} a^{2} \operatorname{Vol}_{g}(\mathcal{M}) t^{2}-\frac{\mu}{2^{*}} a^{2^{*}}\|K\|_{1} t^{2^{*}}-\lambda \int_{\mathcal{M}} F(t a) d \sigma_{g} \\
& \leq\left(\frac{1}{2}-\lambda c\right) a^{2} \operatorname{Vol}_{g}(\mathcal{M}) t^{2}-\frac{\mu}{2^{*}} a^{2^{*}}\|K\|_{1} t^{2^{*}} \\
& <0
\end{aligned}
$$

for big enough $c>0$. As a result, 0 is not a local minimizer of $\mathcal{E}_{\lambda, \mu}$ and $w_{0, \mu, \lambda}$ is not the null function.

Remark 3.4. It is worth noticing that assumption $\left(f_{2}\right)$ comes into play only at the end of the proof of Theorem 3.1, to prevent 0 from being a local minimum point of the energy. Therefore it can be replaced by any other assumption, compatible with $\left(f_{1}\right)$, which ensures this fact. For instance, if $f(0) \neq 0$, then it is easy to verify that 0 is not a solution to $\left(P_{\lambda, \mu}\right)$ and so $w_{0, \lambda, \mu}$ is nonzero. Another hypothesis, more restrictive than $\left(f_{2}\right)$, which serves our purpose is

$$
\liminf _{t \rightarrow 0^{+}} \frac{F(t)}{t^{b}}>0, \quad \text { for some } b \in(1,2) .
$$

The next result shows that, by lightly strengthening the assumptions on $f$, one can determine the sign of the local minimizer.

Theorem 3.5. Let $f: \mathbb{R} \rightarrow \mathbb{R}$ satisfy $\left(f_{1}\right),\left(f_{2}\right)$ and $f(0)=0$. Then for every $\mu>0$ and sufficiently small $\lambda$, problem $\left(P_{\lambda, \mu}\right)$ admits a nonnegative solution $w_{0, \mu, \lambda}^{\star}$ in $H_{\alpha}^{2}(\mathcal{M})$.

Proof. Associated with the function

$$
F_{+}(t):=\int_{0}^{t} f_{+}(\tau) d \tau
$$

for every $t \in \mathbb{R}$, where

$$
f_{+}(\tau):= \begin{cases}f(\tau) & \text { if } \tau>0 \\ 0 & \text { if } \tau \leq 0\end{cases}
$$

let us introduce the functional $\mathcal{E}_{\lambda, \mu}^{+}: H_{\alpha}^{2}(\mathcal{M}) \rightarrow \mathbb{R}$ given by

$$
\mathcal{E}_{\lambda, \mu}^{+}(w):=\frac{1}{2}\|w\|^{2}-\frac{\mu}{2^{*}} \int_{\mathcal{M}} K(\sigma) w_{+}(\sigma)^{2^{*}} d \sigma_{g}-\lambda \int_{\mathcal{M}} F_{+}(w(\sigma)) d \sigma_{g},
$$

where $u_{+}:=\max \{u, 0\}$, for any $w \in H_{\alpha}^{2}(\mathcal{M})$.

It is a simple matter to check that $\mathcal{E}_{\lambda, \mu}^{+}$is well-defined and Gâteaux-differentiable on $H_{\alpha}^{2}(\mathcal{M})$, so by Theorem 3.1 , for every $\mu>0$ and $\lambda$ sufficiently small, it admits a critical point $w_{0, \mu, \lambda}^{\star} \in H_{\alpha}^{2}(\mathcal{M})$.

We claim that $w_{0, \mu, \lambda}^{\star}$ is nonnegative on $\mathcal{M}$. Indeed, since $w_{0, \mu, \lambda}^{\star} \in H_{\alpha}^{2}(\mathcal{M})$, it follows that $\left(w_{0, \mu, \lambda}^{\star}\right)_{-}:=\max \left\{-w_{0, \mu, \lambda}^{\star}, 0\right\}$ belongs to $H_{\alpha}^{2}(\mathcal{M})$ as well. So, taking also into account the 
relationship

$$
\begin{aligned}
\left\langle w_{0, \mu, \lambda}^{\star},\left(w_{0, \mu, \lambda}^{\star}\right)_{-}\right\rangle= & \int_{\mathcal{M}}\left\langle\nabla w_{0, \mu, \lambda}^{\star}(\sigma), \nabla\left(w_{0, \mu, \lambda}^{\star}\right)_{-}(\sigma)\right\rangle_{g} d \sigma_{g} \\
& +\int_{\mathcal{M}} \alpha(\sigma)\left\langle w_{0, \mu, \lambda}^{\star}(\sigma),\left(w_{0, \mu, \lambda}^{\star}\right)_{-}(\sigma)\right\rangle_{g} d \sigma_{g} \\
= & -\int_{\mathcal{M}}\left(\left|\nabla\left(w_{0, \mu, \lambda}^{\star}\right)_{-}(\sigma)\right|^{2}+\alpha(\sigma)\left(w_{0, \mu, \lambda}^{\star}\right)_{-}(\sigma)^{2}\right) d \sigma_{g},
\end{aligned}
$$

we get

$$
\begin{aligned}
-\left\|\left(w_{0, \mu, \lambda}^{\star}\right)_{-}\right\|^{2}= & \left\langle w_{0, \mu, \lambda}^{\star},\left(w_{0, \mu, \lambda}^{\star}\right)_{-}\right\rangle \\
= & \mu \int_{\mathcal{M}} K(\sigma)\left(w_{0, \mu, \lambda}^{\star}\right)+(\sigma)^{2^{*}-1}\left(w_{0, \mu, \lambda}^{\star}\right)_{-}(\sigma) d \sigma_{g} \\
& +\lambda \int_{\mathcal{M}} f_{+}\left(w_{0, \mu, \lambda}^{\star}(\sigma)\right)\left(w_{0, \mu, \lambda}^{\star}\right)_{-}(\sigma) d \sigma_{g} \\
= & \lambda \int_{\mathcal{M}} f_{+}\left(w_{0, \mu, \lambda}^{\star}(\sigma)\right)\left(w_{0, \mu, \lambda}^{\star}\right)_{-}(\sigma) d \sigma_{g} \\
= & 0 .
\end{aligned}
$$

As a result, $\left\|\left(w_{0, \mu, \lambda}^{\star}\right)_{-}\right\|=0$ and hence $w_{0, \mu, \lambda}^{\star} \geq 0$ a.e. on $\mathcal{M}$.

Remark 3.6. In the same way, if $f: \mathbb{R} \rightarrow \mathbb{R}$ satisfies $\left(f_{1}\right)$,

$\left(f_{2}^{\prime}\right) \liminf _{t \rightarrow 0^{-}} \frac{F(t)}{t^{2}}=+\infty$

and $f(0)=0$, then, arguing as in Theorem 3.5, one can study the existence of a nonpositive solution to $\left(P_{\lambda, \mu}\right)$. It suffices to consider the functional

$$
\mathcal{E}_{\lambda, \mu}^{-}(w):=\frac{1}{2}\|w\|^{2}-\frac{\mu}{2^{*}} \int_{\mathcal{M}} K(\sigma) w_{-}(\sigma)^{2^{*}} d \sigma_{g}-\lambda \int_{\mathcal{M}} F_{-}(w(\sigma)) d \sigma_{g},
$$

where

$$
F_{-}(t):=\int_{0}^{t} f_{-}(\tau) d \tau
$$

for every $t \in \mathbb{R}$, and

$$
f_{-}(\tau):= \begin{cases}f(\tau) & \text { if } \tau<0 \\ 0 & \text { if } \tau \geq 0\end{cases}
$$

The underlying idea of the proof of Theorem 3.1 remains valid when adding a term singular at zero, i.e. to treat the following singular variant of problem $\left(P_{\lambda, \mu}\right)$ :

$$
\left(P_{\lambda, \mu}^{\star}\right) \quad\left\{\begin{array}{l}
-\Delta_{g} w+\alpha(\sigma) w=\mu K(\sigma) w^{\frac{d+2}{d-2}}+\lambda\left(w^{r-1}+f(w)\right), \quad \sigma \in \mathcal{M} \\
w \in H_{\alpha}^{2}(\mathcal{M}), \quad w>0 \text { in } \mathcal{M},
\end{array}\right.
$$

where $r \in(0,1)$ and $f:[0,+\infty) \rightarrow[0,+\infty)$ is continuous and subcritical. In this context a weak solution to $\left(P_{\lambda, \mu}^{\star}\right)$ is meant to be any $w \in H_{\alpha}^{2}(\mathcal{M})$ such that $w>0$ a.e. in $\mathcal{M}$, $w^{r-1} z \in L^{1}(\mathcal{M})$ for any $z \in H_{\alpha}^{2}(\mathcal{M})$ and

$$
\langle w, z\rangle-\mu \int_{\mathcal{M}} K(\sigma) w^{\frac{d+2}{d-2}} z d \sigma_{g}-\lambda \int_{\mathcal{M}}\left(w^{r-1}+f(w)\right) z d \sigma_{g}=0
$$


for each $z \in H_{\alpha}^{2}(\mathcal{M})$. The energy naturally associated with $\left(P_{\lambda, \mu}^{\star}\right)$ is

$$
\begin{aligned}
\mathcal{E}_{\lambda, \mu}(w):= & \frac{1}{2}\|w\|^{2}-\frac{\mu}{2^{*}} \int_{\mathcal{M}} K(\sigma)\left(w^{+}\right)^{2^{*}} d \sigma_{g} \\
& -\frac{\lambda}{r} \int_{\mathcal{M}}\left(w^{+}\right)^{r} d \sigma_{g}-\lambda \int_{\mathcal{M}} F\left(w^{+}\right) d \sigma_{g},
\end{aligned}
$$

for all $w \in H_{\alpha}^{2}(\mathcal{M})$. As before, for any positive $\mu$, the range of $\lambda$ for which $\left(P_{\lambda, \mu}^{\star}\right)$ admits nontrivial solutions is strictly related to the maximum of an auxiliary rational function similar to (3.1).

Theorem 3.7. Let $f:[0,+\infty) \rightarrow[0,+\infty)$ be a continuous function for which

$\left(f_{1}^{\prime}\right)$ there exist $a_{1}, a_{2} \geq 0$ and $q \in\left[1,2^{*}\right)$ such that

$$
f(t) \leq a_{1}+a_{2} t^{q-1} \quad \text { for all } t \geq 0
$$

For any $\mu>0$ let $m_{\mu}:[0,+\infty) \rightarrow \mathbb{R}$ be the function defined by

$$
m_{\mu}(t):=\frac{t^{2-r}-\mu c_{2^{*}}^{2^{*}}\|K\|_{\infty} t^{2^{*}-r}}{c_{2^{*}}^{r} \operatorname{Vol}_{g}(\mathcal{M})^{\frac{2^{*}-r}{2^{*}}}+a_{1} c_{2^{*}} \operatorname{Vol}_{g}(\mathcal{M})^{\frac{2^{*}-1}{2^{*}}} t^{1-r}+a_{2} c_{2^{*}}^{q} \operatorname{Vol}_{g}(\mathcal{M})^{\frac{2^{*}-q}{2^{*}}} t^{q-r}},
$$

for avery $t \geq 0$.

Then for every $\mu>0$ there exists an open interval

$$
\Lambda_{\mu} \subseteq\left(0, \max _{[0,+\infty)} m_{\mu}\right)
$$

such that, for every $\lambda \in \Lambda_{\mu},\left(P_{\lambda, \mu}^{\star}\right)$ admits a nontrivial weak solution $\tilde{w}_{0, \lambda, \mu} \in H_{\alpha}^{2}(\mathcal{M})$.

Proof. It is clear that for all $\lambda, \mu>0$ the functionals

$$
\begin{aligned}
& H_{\alpha}^{2}(\mathcal{M}) \ni w \mapsto \frac{1}{2}\|w\|^{2}-\frac{\mu}{2^{*}} \int_{\mathcal{M}} K(\sigma)\left(w^{+}\right)^{2^{*}} d \sigma_{g} \\
& H_{\alpha}^{2}(\mathcal{M}) \ni w \mapsto \frac{\mu}{2^{*}} \int_{\mathcal{M}} K(\sigma)\left(w^{+}\right)^{2^{*}} d \sigma_{g}+\frac{\lambda}{r} \int_{\mathcal{M}}\left(w^{+}\right)^{r} d \sigma_{g}+\lambda \int_{\mathcal{M}} F\left(w^{+}\right) d \sigma_{g}
\end{aligned}
$$

satisfy Lemmas 3.2 and 3.3, respectively.

Arguing exactly as in Theorem 3.1, we deduce that the functional $\mathcal{E}_{\lambda, \mu}$ defined by (3.14), attains a minimum $\tilde{w}_{0, \lambda, \mu}$ on a sufficiently small ball

$$
B_{c}\left(0, \varrho_{0, \mu, \lambda}\right) \subset H_{\alpha}^{2}(\mathcal{M})
$$

and such a minimum is not identically zero. Indeed, fixing $w \in H_{\alpha}^{2}(\mathcal{M}), w>0$ on $\mathcal{M}$, if $t>0$ one has

$$
\begin{aligned}
\mathcal{E}_{\lambda, \mu}(t w) \leq & \frac{1}{2}\|w\|^{2} t^{2}-\frac{\mu}{2^{*}} \operatorname{essinf} K\|w\|_{2^{*}}^{2^{*}} t^{2^{*}}+\lambda a_{1}\|w\|_{1} t \\
& +\frac{\lambda a_{2}}{q}\|w\|_{q}^{q} t^{q}-\frac{\lambda}{r} \int_{\mathcal{M}}|w|^{r} d \sigma_{g} t^{r}
\end{aligned}
$$

and hence $\mathcal{E}_{\lambda, \mu}(t w)$ is negative for all small enough $t$. Arguments similar to those of [10, Theorem 4.1] finally show that $\tilde{w}_{0, \lambda, \mu}$ weakly solves $\left(P_{\lambda, \mu}^{\star}\right)$. 


\section{Existence of MP-Type solutions}

In this section we establish another existence result for $\left(P_{\lambda, \mu}\right)$, this time of the mountain pass type. The abstract tool we rely upon is a version of the mountain pass theorem without the Palais-Smale condition ([5, Theorem 2.2]) and, as usual in the treatment of critical problems via this approach, the crucial point is the relationship between the mountain pass level and the constant $S$ defined by (2.6).

Theorem 4.1. Let $f: \mathbb{R} \rightarrow \mathbb{R}$ be a continuous function satisfying $\left(f_{1}\right)$ and

$\left(f_{3}\right) \lim _{t \rightarrow 0} \frac{f(t)}{t}=0$,

and let $\lambda, \mu>0$. Furthermore, assume that there exists $w^{\star} \in H_{\alpha}^{2}(\mathcal{M}) \backslash\{0\}, w^{\star} \geq 0$ a.e. in $\mathcal{M}$, such that

$$
\left(H_{1}\right) \sup _{\tau \geq 0} \mathcal{E}_{\lambda, \mu}\left(\tau w^{\star}\right)<\frac{S^{\frac{d}{2}}}{d\left(\mu\|K\|_{\infty}\right)^{\frac{d-2}{2}}} .
$$

Then problem $\left(P_{\lambda, \mu}\right)$ admits a nontrivial solution.

Proof. Let us show first that $\mathcal{E}_{\lambda, \mu}$ possesses the geometry required by Theorem 2.2 of [5].

Claim 4.1. There exist $\varrho, \beta>0$ such that for any $w \in \partial B(0, \varrho)$ one has $\mathcal{E}_{\lambda, \mu}(w) \geq \beta$.

Fix $\varepsilon \in\left(0, \lambda^{-1} c_{2}^{-2}\right)$. Thanks to $\left(f_{3}\right)$, there exists $\delta_{\epsilon}>0$ so that

$$
|f(t)| \leq \varepsilon|t|
$$

for every $t \in\left(-\delta_{\varepsilon}, \delta_{\varepsilon}\right)$. On the other hand, $\left(f_{1}\right)$ allows us to deduce that

$$
\lim _{|t| \rightarrow+\infty} \frac{|f(t)|}{|t|^{2}-1}=0
$$

and therefore there exists $a_{\varepsilon}>0$ such that

$$
|f(t)| \leq \varepsilon|t|^{2^{*}-1}+a_{\varepsilon}
$$

for all $t \in \mathbb{R}$ and, consequently,

$$
|f(t)| \leq \varepsilon|t|^{2^{*}-1}+a_{\varepsilon} \frac{|t|^{2^{*}-1}}{\delta_{\varepsilon}^{2^{*}-1}}=b_{\varepsilon}|t|^{2^{*}-1}
$$

for every $t \in \mathbb{R} \backslash\left(-\delta_{\varepsilon}, \delta_{\varepsilon}\right)$. Hence we get

$$
|F(t)| \leq \frac{\varepsilon}{2} t^{2}+\frac{b_{\varepsilon}}{2^{*}}|t|^{2^{*}}
$$

for every $t \in \mathbb{R}$. In the light of the above estimates, for every $w \in H_{\alpha}^{2}(\mathcal{M})$ we obtain

$$
\begin{aligned}
\mathcal{E}_{\lambda, \mu}(w) & \geq \frac{1}{2}\|w\|^{2}-\frac{\mu}{2^{*}}\|K\|_{\infty}\|w\|_{2^{*}}^{2^{*}}-\frac{\lambda \varepsilon}{2}\|w\|_{2}^{2}-\frac{\lambda b_{\varepsilon}}{2^{*}}\|w\|_{2^{*}}^{2^{*}} \\
& \geq\left(\frac{1-\lambda \varepsilon c_{2}^{2}}{2}\right)\|w\|^{2}-\left(\frac{\mu\|K\|_{\infty}+\lambda b_{\varepsilon}}{2^{*}}\right) c_{2^{*}}^{2^{*}}\|w\|^{2^{*}} .
\end{aligned}
$$

Thanks to the choice of $\varepsilon$, for suitable $a, b>0$ we have

$$
\mathcal{E}_{\lambda, \mu}(w) \geq a\|w\|^{2}\left(1-b\|w\|^{\frac{4}{d-2}}\right)
$$

and so, by choosing $\varrho \in\left(0, b^{\frac{2-d}{4}}\right)$, we get

$$
\inf _{\partial B(0, \varrho)} \mathcal{E} \geq a \varrho^{2}\left(1-b \varrho^{\frac{4}{d-2}}\right)=: \beta>0
$$

and the conclusion is achieved. 
Claim 4.2. There exists $w_{1} \in H_{\alpha}^{2}(\mathcal{M})$ such that $w_{1} \geq 0$ a.e. in $\mathcal{M},\left\|w_{1}\right\|>\varrho$ and $\mathcal{E}_{\lambda, \mu}\left(w_{1}\right)<\beta$, where $\varrho, \beta$ have the same meaning as in Claim 4.1.

If $\tau>0$ and $w^{\star}$ is the function defined in $\left(H_{1}\right)$, we get

$$
\begin{aligned}
\mathcal{E}_{\lambda, \mu}\left(\frac{\tau w^{\star}}{\left\|w^{\star}\right\|}\right)= & \frac{1}{2} \tau^{2}-\frac{\mu}{2^{*}\left\|w^{\star}\right\|^{2^{*}}} \int_{\mathcal{M}} K(\sigma)\left|\tau w^{\star}\right|^{2^{*}} d \sigma_{g}-\lambda \int_{\mathcal{M}} F\left(\frac{\tau w^{\star}}{\left\|w^{\star}\right\|}\right) d \sigma_{g} \\
\leq & \frac{1}{2} \tau^{2}-\frac{\mu}{2^{*}\left\|w^{\star}\right\|^{2^{*}}} \operatorname{essinf} K\left\|w^{\star}\right\|_{2^{*}}^{2^{*}} \tau^{2^{*}} \\
& +\lambda a_{1} \frac{\left\|w^{\star}\right\|_{1}}{\left\|w^{\star}\right\|} \tau+\frac{\lambda a_{2}}{q} \cdot \frac{\left\|w^{\star}\right\|_{q}^{q}}{\left\|w^{\star}\right\|^{q}} \tau^{q}
\end{aligned}
$$

and therefore $\mathcal{E}_{\lambda, \mu}\left(\tau w^{\star} /\left\|w^{\star}\right\|\right) \rightarrow-\infty$ as $\tau \rightarrow+\infty$. So it suffices to pick $w_{1}:=\tau_{0} w^{\star} /\left\|w^{\star}\right\|$, with large enough $\tau_{0}>0$, to obtain the claim.

Now, set

$$
c:=\inf _{\gamma \in \Gamma} \sup _{t \in[0,1]} \mathcal{E}_{\lambda, \mu}(\gamma(t))
$$

where

$$
\Gamma:=\left\{\gamma \in C^{0}\left([0,1], H_{\alpha}^{2}(\mathcal{M})\right): \gamma(0)=0 \text { and } \gamma(1)=w_{1}\right\} .
$$

For any $\gamma \in \Gamma$, the function $t \mapsto\|\gamma(t)\|$ is continuous on $[0,1]$, so by the intermediate value theorem there exists $\bar{t} \in(0,1)$ such that $\|\gamma(\bar{t})\|=\varrho$. As a result

$$
\sup _{t \in[0,1]} \mathcal{E}_{\lambda, \mu}(\gamma(t)) \geq \mathcal{E}_{\lambda, \mu}(\gamma(\bar{t})) \geq \inf _{\partial B(0, \varrho)} \mathcal{E}_{\lambda, \mu}
$$

which implies $c \geq \beta$. Moreover, thanks to $\left(H_{1}\right)$ and the fact that the map $t \mapsto w_{1} t, t \in[0,1]$, is an element of $\Gamma$, one has the estimate

$$
c \leq \sup _{\tau \geq 0} \mathcal{E}_{\lambda, \mu}\left(\tau w_{1}\right)<\frac{S^{\frac{d}{2}}}{d\left(\mu\|K\|_{\infty}\right)^{\frac{d-2}{2}}} .
$$

Next, due to Theorem 2.2 of [5], there exists a sequence $\left\{w_{j}\right\}_{j \in \mathbb{N}} \subset H_{\alpha}^{2}(\mathcal{M})$ satisfying

$$
\mathcal{E}_{\lambda, \mu}\left(w_{j}\right) \rightarrow c, \quad \mathcal{E}_{\lambda, \mu}^{\prime}\left(w_{j}\right) \rightarrow 0, \quad \text { as } j \rightarrow \infty .
$$

Claim 4.3. The sequence $\left\{w_{j}\right\}_{j \in \mathbb{N}}$ is bounded in $H_{\alpha}^{2}(\mathcal{M})$.

For large enough $j$, using also (4.2) with $\varepsilon \in\left(0, \mu \operatorname{essinf}_{\mathcal{M}} K / \lambda(d-1)\right)$, we have

$$
\begin{aligned}
c+1+\left\|w_{j}\right\| & \geq \mathcal{E}_{\lambda, \mu}\left(w_{j}\right)-\frac{1}{2} \mathcal{E}_{\lambda, \mu}^{\prime}\left(w_{j}\right)\left(w_{j}\right) \\
& =\frac{\mu}{d} \int_{\mathcal{M}} K(\sigma)\left|w_{j}\right|^{2^{*}} d \sigma_{g}-\lambda \int_{\mathcal{M}} F\left(w_{j}\right) d \sigma_{g}+\frac{\lambda}{2} \int_{\mathcal{M}} f\left(w_{j}\right) w_{j} d \sigma_{g} \\
& \geq \frac{\mu}{d} \operatorname{essinf} K\left\|w_{j}\right\|_{2^{*}}^{2^{*}}-\left(\frac{\lambda \varepsilon}{2^{*}}+\frac{\lambda \varepsilon}{2}\right)\left\|w_{j}\right\|_{2^{*}}^{2^{*}}-\frac{3}{2} \lambda a_{\varepsilon}\left\|w_{j}\right\|_{1} \\
& \geq\left(\frac{2 \mu \operatorname{essinf}_{\mathcal{M}} K-\lambda \varepsilon(d-2)-\lambda \varepsilon d}{2 d}\right)\left\|w_{j}\right\|_{2^{*}}^{2^{*}}-\frac{3}{2} \lambda a_{\varepsilon} c_{1}\left\|w_{j}\right\|,
\end{aligned}
$$

and therefore, for some $\kappa_{1}>0$,

$$
\left\|w_{j}\right\|_{2^{*}}^{2^{*}} \leq \kappa_{1}\left(1+\left\|w_{j}\right\|\right)
$$


On the other hand, again for large values of $j$, taking (4.8) into account one has

$$
\begin{aligned}
c+1 & \geq \mathcal{E}_{\lambda, \mu}\left(w_{j}\right) \geq \frac{1}{2}\left\|w_{j}\right\|^{2}-\left(\frac{\mu}{2^{*}}\|K\|_{\infty}+\frac{\lambda \varepsilon}{2^{*}}\right)\left\|w_{j}\right\|_{2^{*}}^{2^{*}}-\lambda a_{\varepsilon} c_{1}\left\|w_{j}\right\| \\
& \geq \frac{1}{2}\left\|w_{j}\right\|^{2}-\left(\frac{\mu\|K\|_{\infty}+\lambda \varepsilon(d-2)}{2 d}\right) \kappa_{1}\left(1+\left\|w_{j}\right\|\right)-\lambda a_{\varepsilon} c_{1}\left\|w_{j}\right\|,
\end{aligned}
$$

which forces

$$
\left\|w_{j}\right\|^{2} \leq \kappa_{2}\left(1+\left\|w_{j}\right\|\right)
$$

for some $\kappa_{2}>0$, and hence the boundedness of $\left\{w_{j}\right\}_{j \in \mathbb{N}}$.

As a consequence of the previous claim, there exists $w_{\infty} \in H_{\alpha}^{2}(\mathcal{M})$ such that, up to a subsequence (still denoted by $\left\{w_{j}\right\}_{j \in \mathbb{N}}$ ), $w_{j} \rightarrow w_{\infty}$, that is

$$
\left\langle w_{j}, z\right\rangle \rightarrow\left\langle w_{\infty}, z\right\rangle \quad \text { as } j \rightarrow \infty
$$

for any $z \in H_{\alpha}^{2}(\mathcal{M})$. Moreover, since $\left\{w_{j}\right\}_{j \in \mathbb{N}}$ is bounded in $L^{2^{*}}(\mathcal{M})$ as well, passing to a further subsequence, we get the validity of the following convergences:

$$
\begin{aligned}
& w_{j} \rightarrow w_{\infty} \quad \text { in } L^{2^{*}}(\mathcal{M}) \\
& w_{j} \rightarrow w_{\infty} \quad \text { in } L^{p}(\mathcal{M}), \quad p \in\left[1,2^{*}\right) \\
& w_{j} \rightarrow w_{\infty} \quad \text { a.e. in } \mathcal{M} \\
& \left|w_{j}\right|^{\frac{4}{d-2}} w_{j} \rightarrow\left|w_{\infty}\right|^{\frac{4}{d-2}} w_{\infty} \quad \text { in } L^{\frac{2 d}{d+2}}(\mathcal{M}),
\end{aligned}
$$

as $j \rightarrow \infty$. Moreover, thanks to (4.2) and (4.10), we easily obtain

$$
\begin{array}{ll}
f\left(w_{j}(\cdot)\right) \rightarrow f\left(w_{\infty}(\cdot)\right) & \text { a.e. in } \mathcal{M} \\
f\left(w_{j}(\cdot)\right) \rightarrow f\left(w_{\infty}(\cdot)\right) & \text { in } L^{\frac{2 d}{d+2}}(\mathcal{M}),
\end{array}
$$

as $j \rightarrow \infty$. As a result,

$$
\int_{\mathcal{M}} f\left(w_{j}\right) z d \sigma_{g} \rightarrow \int_{\mathcal{M}} f\left(w_{\infty}\right) z d \sigma_{g} \quad \text { as } j \rightarrow \infty
$$

for any $z \in L^{2^{*}}(\mathcal{M})$ and, a fortiori,

$$
\int_{\mathcal{M}} f\left(w_{j}\right) z d \sigma_{g} \rightarrow \int_{\mathcal{M}} f\left(w_{\infty}\right) z d \sigma_{g} \quad \text { as } j \rightarrow \infty
$$

for any $z \in H_{\alpha}^{2}(\mathcal{M})$. Now, recalling that

$$
\mathcal{E}_{\lambda, \mu}^{\prime}\left(w_{j}\right)(z)=\left\langle w_{j}, z\right\rangle-\mu \int_{\mathcal{M}} K(\sigma)\left|w_{j}\right|^{\frac{4}{d-2}} w_{j} z d \sigma_{g}-\lambda \int_{\mathcal{M}} f\left(w_{j}\right) z d \sigma_{g},
$$

for any $z \in H_{\alpha}^{2}$, passing to the limit as $j \rightarrow \infty$ in the above equality and taking (4.7), (4.9), (4.10) and (4.11) and the fact that $K \in \Lambda_{+}(\mathcal{M})$ into account, we get

$$
\left\langle w_{\infty}, z\right\rangle-\mu \int_{\mathcal{M}} K(\sigma)\left|w_{\infty}\right|^{\frac{4}{d-2}} w_{\infty} z d \sigma_{g}-\lambda \int_{\mathcal{M}} f\left(w_{\infty}\right) z d \sigma_{g}=0
$$

for any $z \in H_{\alpha}^{2}(\mathcal{M})$, so $w_{\infty}$ is the weak solution to $\left(P_{\lambda, \mu}\right)$ we were looking for.

Arguing by contradiction, finally suppose that $w_{\infty} \equiv 0$ in $\mathcal{M}$. Then, due to (4.2), for any $j \in \mathbb{N}$ and for some $\kappa>0$ we would obtain

$$
\left|\int_{\mathcal{M}} f\left(w_{j}\right) w_{j} d \sigma_{g}\right| \leq \varepsilon\left\|w_{j}\right\|_{2^{*}}^{2^{*}}+a_{\varepsilon}\left\|w_{j}\right\|_{1} \leq \varepsilon \kappa+a_{\varepsilon}\left\|w_{j}\right\|_{1},
$$


and

$$
\left|\int_{\mathcal{M}} F\left(w_{j}\right) d \sigma_{g}\right| \leq \frac{\varepsilon}{2^{*}}\left\|w_{j}\right\|_{2^{*}}^{2^{*}}+a_{\varepsilon}\left\|w_{j}\right\|_{1} \leq \frac{\varepsilon \kappa}{2^{*}}+a_{\varepsilon}\left\|w_{j}\right\|_{1} .
$$

Exploiting (4.10) and taking limsup as $j \rightarrow \infty$ and $\lim$ as $\varepsilon \rightarrow 0$ in the above inequalities one has

$$
\lim _{j \rightarrow \infty} \int_{\mathcal{M}} f\left(w_{j}\right) w_{j} d \sigma_{g}=\lim _{j \rightarrow \infty} \int_{\mathcal{M}} F\left(w_{j}\right) d \sigma_{g}=0
$$

and therefore, since $\mathcal{E}_{\lambda, \mu}^{\prime}\left(w_{j}\right)\left(w_{j}\right) \rightarrow 0$ as $j \rightarrow \infty$,

$$
\left\|w_{j}\right\|^{2}-\mu \int_{\mathcal{M}} K(\sigma)\left|w_{j}\right|^{2^{*}} d \sigma_{g} \rightarrow 0 \quad \text { as } j \rightarrow \infty .
$$

Now, the boundedness of $\left\{\left\|w_{j}\right\|\right\}_{j \in \mathbb{N}}$ in $\mathbb{R}$ implies that, up to a subsequence, there exists $L \in[0,+\infty)$ such that

$$
\left\|w_{j}\right\|^{2} \rightarrow L
$$

and so

$$
\int_{\mathcal{M}} K(\sigma)\left|w_{j}\right|^{2^{*}} d \sigma_{g} \rightarrow \frac{L}{\mu}
$$

as $j \rightarrow+\infty$. Recalling that

$$
\begin{aligned}
\mathcal{E}_{\lambda, \mu}\left(w_{j}\right)= & \frac{1}{2}\left\|w_{j}\right\|^{2}-\frac{\mu}{2^{*}} \int_{\mathcal{M}} K(\sigma)\left|w_{j}\right|^{2^{*}} d \sigma_{g} \\
& -\lambda \int_{\mathcal{M}} F\left(w_{j}\right) d \sigma_{g} \rightarrow c \text { as } j \rightarrow \infty,
\end{aligned}
$$

it follows on account of (4.12), (4.14) and (4.15), that

$$
c=\left(\frac{1}{2}-\frac{1}{2^{*}}\right) L=\frac{L}{d},
$$

which due to $c \geq \beta>0$, forces $L>0$. On the other hand, from (4.15) we get

$$
\lim _{j \rightarrow \infty} \int_{\mathcal{M}}\left|w_{j}\right|^{2^{*}} d \sigma_{g} \geq \frac{L}{\mu\|K\|_{\infty}}
$$

and moreover

$$
\left\|w_{j}\right\|^{2} \geq S\left\|w_{j}\right\|_{2^{*}}^{2}
$$

so that, passing to the limit as $j \rightarrow \infty$ in the above relationship and taking (4.14) and (4.17) into account, we get

$$
L \geq S\left(\frac{L}{\mu\|K\|_{\infty}}\right)^{\frac{2}{2^{*}}},
$$

which combined with (4.16), gives

$$
c \geq \frac{S^{\frac{2^{*}}{2^{*}-2}}}{d\left(\mu\|K\|_{\infty}\right)^{\frac{2}{2^{*}-2}}}=\frac{S^{\frac{d}{2}}}{d\left(\mu\|K\|_{\infty}\right)^{\frac{d-2}{2}} .}
$$

This contradicts (4.6) and therefore $w_{\infty} \not \equiv 0$ in $\mathcal{M}$. The proof is hence complete. 


\section{The Case of the Sphere: applications to the Emden-Fowler equation}

As already explained in the introduction, an interesting case of problem $\left(P_{\lambda, \mu}\right)$ is

$$
\begin{aligned}
& -\Delta_{h} w+s(1-s-d) w \\
& \quad=\mu K(\sigma)|w|^{\frac{4}{d-2}} w+\lambda f(w), \quad \sigma \in \mathbb{S}^{d}, w \in H_{1}^{2}\left(\mathbb{S}^{d}\right)
\end{aligned}
$$

where $\mathbb{S}^{d}$ is the unit sphere in $\mathbb{R}^{d+1}, h$ is the standard metric induced by the embedding $\mathbb{S}^{d} \hookrightarrow \mathbb{R}^{d+1}, s \in \mathbb{R}$ is a constant related to $d$ by the relationship $1-d<s<0$, and $\Delta_{h}$ is the Laplace-Beltrami operator on $\left(\mathbb{S}^{d}, h\right)$.

Existence results for $\left(\widetilde{P}_{\lambda, \mu}\right)$, via a suitable change of coordinates, produce the existence of solutions to the following Emden-Fowler equation

$$
\begin{aligned}
\left(\bar{P}_{\lambda, \mu}\right) \quad-\Delta u= & \mu|x|^{\frac{2(2-2 s-d)}{d-2}} K\left(\frac{x}{|x|}\right)|u|^{\frac{4}{d-2}} u \\
& +\lambda|x|^{s-2} f\left(\frac{u}{|x|^{s}}\right), \quad x \in \mathbb{R}^{d+1} \backslash\{0\} .
\end{aligned}
$$

Indeed, let us seek solutions to $\left(\bar{P}_{\lambda, \mu}\right)$ of the form

$$
u(x)=r^{s} w(\sigma),
$$

where $(r, \sigma):=(|x|, x /|x|) \in(0,+\infty) \times \mathbb{S}^{d}$ are the spherical coordinates in $\mathbb{R}^{d+1} \backslash\{0\}$ and $w$ a smooth function defined on $\mathbb{S}^{d}$. Via (5.1) and taking into account that

$$
\begin{aligned}
\Delta u & =r^{-d} \frac{\partial}{\partial r}\left(r^{d} \frac{\partial}{\partial r}\left(r^{s} w\right)\right)+r^{s-2} \Delta_{h} w \\
& =\left[s(d+s-1)+\Delta_{h} w\right] r^{s-2},
\end{aligned}
$$

it is easily seen that the study of problem $\left(\widetilde{P}_{\lambda, \mu}\right)$ can be successfully applied to treat problem $\left(\bar{P}_{\lambda, \mu}\right)$.

Theorem 5.1. Let $d, s \in \mathbb{R}$ be such that $1-d<s<0, K \in \Lambda_{+}\left(\mathbb{S}^{d}\right)$ and $f: \mathbb{R} \rightarrow \mathbb{R}$ be a locally Lipschitz continuous function satisfying $\left(f_{1}\right)-\left(f_{2}\right)$. Furthermore, for any $\mu>0$, let $l_{\mu}:[0,+\infty) \rightarrow \mathbb{R}$ be the function defined by

$$
l_{\mu}(t):=\frac{t-\mu c_{2^{*}}^{2^{*}}\|K\|_{\infty} t^{2^{*}-1}}{a_{1} c_{2^{*}} \omega_{d}^{2^{2^{*}}-1}+a_{2} c_{2^{*}}^{q} \omega_{d}^{\frac{2^{*}-q}{2^{*}}} t^{q-1}} \quad \text { for all } t \geq 0 .
$$

Then for every $\mu>0$ there exists an open interval

$$
\Lambda_{\mu} \subseteq\left(0, \max _{[0,+\infty)} l_{\mu}\right)
$$

such that for every $\lambda \in \Lambda_{\mu}$, the problem

$$
\begin{aligned}
\left(\bar{P}_{\lambda, \mu}\right) \quad-\Delta u= & \mu|x|^{\frac{2(2-2 s-d)}{d-2}} K\left(\frac{x}{|x|}\right)|u|^{\frac{4}{d-2}} u \\
& +\lambda|x|^{s-2} f\left(\frac{u}{|x|^{s}}\right), \quad x \in \mathbb{R}^{d+1} \backslash\{0\}
\end{aligned}
$$

admits a nontrivial solution. 
Proof. Let us appeal to Theorem 3.1 by choosing $(\mathcal{M}, g)=\left(\mathbb{S}^{d}, h\right)$ and $\alpha(\sigma):=s(1-s-d)$ for every $\sigma \in \mathbb{S}^{d}$. Thanks to the relationship between $d$ and $s$, clearly $\alpha \in \Lambda_{+}\left(\mathbb{S}^{d}\right)$. So the problem

$$
\begin{aligned}
& -\Delta_{h} w+s(1-s-d) w \\
& \quad=\mu K(\sigma)|w|^{\frac{4}{d-2}} w+\lambda f(w), \quad \sigma \in \mathbb{S}^{d}, w \in H_{1}^{2}\left(\mathbb{S}^{d}\right)
\end{aligned}
$$

admits at least a nontrivial solution $w_{0, \mu, \lambda} \in H_{\alpha}^{2}\left(\mathbb{S}^{d}\right)$.

But, due to (5.1), $u(x)=|x|^{s} w_{0, \mu, \lambda}(x /|x|)$ is a nontrivial solution to $\left(\bar{P}_{\lambda, \mu}\right)$ and the proof is completed.

In the same manner, we can obtain the following

Theorem 5.2. Let $d, s \in \mathbb{R}$ be such that $1-d<s<0, K \in \Lambda_{+}\left(\mathbb{S}^{d}\right)$ and $f: \mathbb{R} \rightarrow \mathbb{R}$ be a locally Lipschitz continuous function satisfying $\left(f_{1}\right)-\left(f_{2}\right)$ and $f(0)=0$. Then for every $\mu>0$ and sufficiently small $\lambda$, problem $\left(\bar{P}_{\lambda, \mu}\right)$ admits a nonnegative solution.

and

Theorem 5.3. Let $d, s \in \mathbb{R}$ be such that $1-d<s<0, K \in \Lambda_{+}\left(\mathbb{S}^{d}\right)$. Furthermore, let $r \in(0,1)$ and $f:[0,+\infty) \rightarrow[0,+\infty)$ be a locally Lipschitz continuous function for which

$\left(f_{1}^{\prime}\right)$ there exist $a_{1}, a_{2} \geq 0$ and $q \in\left[1,2^{*}\right)$ such that

$$
f(t) \leq a_{1}+a_{2} t^{q-1} \quad \text { for all } t \geq 0 .
$$

For any $\mu>0$ let $m_{\mu}:[0,+\infty) \rightarrow \mathbb{R}$ be the function defined by

$$
m_{\mu}(t):=\frac{t^{2-r}-\mu c_{2^{*}}^{2^{*}}\|K\|_{\infty} t^{2^{*}-r}}{c_{2^{*}}^{r} \omega_{d}^{\frac{2^{*}-r}{2^{*}}}+a_{1} c_{2^{*}} \omega_{d}^{\frac{2^{*}-1}{2^{*}}} t^{1-r}+a_{2} c_{2^{*}}^{q} \omega_{d}^{\frac{2^{*}-q}{2^{*}}} t^{q-r}} \quad \text { for all } t \geq 0 .
$$

Then for every $\mu>0$ there exists an open interval

$$
\Lambda_{\mu} \subseteq\left(0, \max _{[0,+\infty)} m_{\mu}\right)
$$

such that, for every $\lambda \in \Lambda_{\mu}$, the problem

$$
\left\{\begin{array}{l}
-\Delta_{h} w+s(1-s-d) w=\mu K(\sigma) w^{\frac{d+2}{d-2}}+\lambda\left(w^{r-1}+f(w)\right), \quad \sigma \in \mathbb{S}^{d} \\
w \in H_{1}^{2}\left(\mathbb{S}^{d}\right), \quad w>0 \text { in } \mathbb{S}^{d},
\end{array}\right.
$$

admits a solution.

\section{ACKNOWLEDGEMENTS}

The research was supported by the Slovenian Research Agency grants P1-0292, N1-0114, N1-0083, N1-0064, and J1-8131, the Italian MIUR project Variational methods, with applications to problems in mathematical physics and geometry (2015KB9WPT 009), and the INdAM-GNAMPA Project 2017 Metodi variazionali per fenomeni non-locali. The authors thank the referees for comments and suggestions. 


\section{REFERENCES}

[1] G. Albanese and M. Rigoli, Lichnerowicz-type equations on complete manifolds, Adv. Nonlinear Anal. 5 (2016), no. 3, 223-250.

[2] T. Aubin, Équations différentielles non linéaires et problème de Yamabe concernant la courbure scalaire, J. Math. Pures Appl. 55 (1976), 269-296.

[3] T. Aubin, Some Nonlinear Problems in Riemannian Geometry, Springer Monographs in Mathematics, Springer, Berlin, (1998).

[4] W. Beckner, Sharp Sobolev inequalities on the sphere and the Moser-Trudinger inequality, Ann. of Math. 2 (1993), no. 1, 213-242.

[5] H. Brézis and L. Nirenberg, Positive solutions of nonlinear elliptic equations involving critical Sobolev exponents, Comm. Pure Appl. Math. 36 (1983), 437-477.

[6] J. Chabrowski, On multiple solutions for the nonhomogeneous p-Laplacian with a critical Sobolev exponent, Differ. Integr. Equ. 8 (1995), 705-716.

[7] A. Cotsiolis and D. Iliopoulos, Équations elliptiques non linéaires sur $S^{n}$. Le problème de Nirenberg, C. R. Acad. Sci. Paris, Sér. I Math. 313 (1991), 607-609.

[8] A. Cotsiolis and D. Iliopoulos, Équations elliptiques non linéaires à croissance de Sobolev sur-critique, Bull. Sci. Math. 119 (1995), 419-431.

[9] L. Dupaigne, M. Ghergu, and V. Rădulescu, Lane-Emden-Fowler equations with convection and singular potential, J. Math. Pures Appl. 87 (2007), no. 6, 563-581.

[10] F. Faraci and C. Farkas, A quasilinear elliptic problem involving critical Sobolev exponents, Collect. Math. 66 (2015), 243-259.

[11] R. Filippucci, P. Pucci, and F. Robert, On a p-Laplace equation with multiple critical nonlinearities, J. Math. Pures Appl. 91 (2009), no. 2, 156-177.

[12] F. Gazzola and B. Ruf, Lower-order perturbations of critical growth nonlinearities in semilinear elliptic equations, Adv. Differential Equations 2 (1997), 555-572.

[13] H. Hajaiej, G. Molica Bisci, and L. Vilasi, Existence results for a critical fractional equation, Asymptot. Anal. 100 (2016), no. 3-4, 209-225.

[14] E. Hebey, Nonlinear Analysis on Manifolds: Sobolev Spaces and Inequalities, Courant Lecture Notes in Mathematics, Amer. Math. Soc. and Courant Inst. Math. Sci., New York, (1999).

[15] J. L. Kazdan and F. W. Warner, Scalar curvature and conformal deformation of Riemannian structure, J. Differ. Geom. 10 (1975), 113-134.

[16] A. Kristály and V. Rădulescu, Sublinear eigenvalue problems on compact Riemannian manifolds with applications in Emden-Fowler equations, Stud. Math. 191 (2009), 237-246.

[17] A. Kristály, V. Rădulescu, and C. S. Varga, Variational Principles in Mathematical Physics, Geometry, and Economics: Qualitative Analysis of Nonlinear Equations and Unilateral Problems, Encyclopedia of Mathematics and its Applications, Vol. 136, Cambridge University Press, Cambridge, (2010).

[18] P. L. Lions, The concentration-compactness principle in the calculus of variations. The limit case, Rev. Mat. Iberoamericana 1 (1985), 145-201.

[19] G. Molica Bisci and J. Mawhin, A Brezis-Nirenberg type result for a nonlocal fractional operator, J. Lond. Math. Soc. 95 (2017) no. 1, 73-93.

[20] G. Molica Bisci and D. Repovš, Yamabe-type equations on Carnot groups, Potential Anal. 46 (2017), no. 2, 369-383.

[21] G. Molica Bisci and L. Vilasi, Isometry-invariant solutions to a critical problem on non-compact Riemannian manifolds, J. Differential Equations 269 (2020), 5491-5519.

[22] R. Schoen, Conformal deformation of a Riemannian metric to constant scalar curvature, J. Differ. Geom. 20 (1984), 479-495.

[23] M. Squassina, Two solutions for inhomogeneous nonlinear elliptic equations at critical growth, NoDEA Nonlinear Differential Equations Appl. 11 (2004), no. 1, 53-71.

[24] G. Tarantello, On nonhomogeneous elliptic equations involving critical Sobolev exponent, Ann. Inst. H. Poincaré Anal. Non Linéaire 9 (1992), 281-304.

[25] N. Trudinger, Remarks concerning the conformal deformation of Riemannian structures on compact manifolds, Ann. Scuola Norm. Sup. Pisa 22 (1968), 265-274.

[26] J. L. Vázquez and L. Véron, Solutions positives d'équations elliptiques semi-linéaires sur des variétés riemanniennes compactes, C. R. Acad. Sci. Paris, Sér. I Math. 312 (1991), 811-815.

[27] H. Yamabe, On a deformation of Riemannian structures on compact manifolds, Osaka J. Math. 12 (1960), 21-37. 
(G. Molica Bisci) Dipartimento P.A.U., Universit Ã degli Studi Mediterranea di Reggio Calabria, Salita Melissari - Feo di Vito, 89124 Reggio Calabria, Italy \& Institute of Mathematics, Physics and Mechanics, 1000 Ljubljana, Slovenia

Email address: gmolica@unirc.it

(D.D. Repovš) Faculty of Education, and Faculty of Mathematics and Physics, University of Ljubljana \& Institute of Mathematics, Physics and Mechanics, 1000 Ljubluana, Slovenia

Email address: dusan.repovs@guest.arnes.si

(L. Vilasi) Department of Mathematical and Computer Sciences, Physical Sciences and Earth Sciences, University of Messina, Viale F. Stagno D'Alcontres, 31, 98166 Messina, Italy

Email address: lvilasi@unime.it 